\title{
Maternal Stress During Pregnancy Predisposes for Iron Deficiency in Infant Monkeys Impacting Innate Immunity
}

\author{
CHRISTOPHER L. COE, GABRIELE R. LUBACH, AND ELIZABETH A. SHIRTCLIFF
}

Harlow Center for Biological Psychology, University of Wisconsin, Madison, Wisconsin 53715

\begin{abstract}
The influence of maternal stress during pregnancy on the postpartum iron status and immune maturation of infants was investigated in a nonhuman primate model. Forty infant rhesus monkeys were generated from two types of disturbed pregnancies, early or late gestation stress, and compared with 24 undisturbed controls. Prenatal stress increased the prevalence and magnitude of iron deficiency (ID) as the infants' growth-related demands for iron exceeded dietary intake from breast milk. At 4-6 mo of age, the emergence of ID significantly accentuated an effect of prenatal stress on natural killer cell activity, an important component of innate immunity. These findings indicate that maternal stress, especially early in pregnancy, should be added to the list of risk factors that warrant closer scrutiny of hematological profiles in fast-growing babies. (Pediatr Res 61: 520-524, 2007)
\end{abstract}

$I^{\mathrm{D}}$ $D$ is a major nutritional problem affecting 1-2 billion people worldwide (1). The anemia caused by ID is very common in women due to the hematological demands of menstruation and pregnancy and is particularly prevalent in young infants, who also have high iron needs $(2,3)$. To meet the iron requirements of a rapidly growing baby, many mammalian species, including humans, have evolved a two-stage transmission process, providing significant amounts of iron before birth via placental transfer, and the remainder postpartum through breast milk. As a consequence, if iron stores in the form of $\mathrm{Hb}$ and ferritin are low at birth, dietary iron intake from milk will often prove to be inadequate by $2-4$ mo of age (4). These babies are then at greater risk for developing the "early anemia of childhood," which typically becomes evident at $4-8$ mo of age (5).

Although the use of fortified formulas has reduced the prevalence of ID, it continues to impact $3-4 \%$ of $1 \mathrm{y}$ olds in the United States and up to $8-17 \%$ of infants in high-risk populations (6-8). ID is also more likely to occur in babies provided only breast milk past the first year of age or in

Received July 25, 2006; accepted December 13, 2006.

Correspondence: Christopher L. Coe, Ph.D., Harlow Center for Biological Psychology, University of Wisconsin, 22 North Charter Street, Madison, WI 53715; e-mail: ccoe@wisc.edu

Supported by grants from the National Institute of Allergy and Infectious Diseases (AI46521, AI607517) and a Program-Project grant on Brain, Behavior and Early Iron Deficiency from the National Institute of Child Health and Development (P01 HD39386, P.I.: B. Lozoff). EAS is funded on a training grant from the National Institute of Mental Health (T32 MH18931).

DOI: $10.1203 / p d r .0 b 013 e 318045 b e 53$ families that rely on bottle-feeding with cow's milk for economic or cultural reasons (9).

Considerable evidence points to prenatal factors as predisposing and significant contributors to the development of ID (10). Because the bulk of maternal iron is transferred in the last month of pregnancy, babies born premature have low stores, and their iron needs are more likely to exceed the capacity of dietary intake (11). Similarly, small-forgestational age neonates and growth-restricted twins and triplets, who then undergo a compensatory growth spurt, are at higher risk $(12,13)$. Recently, it was discovered that babies gestated by women who had developed pregnancy-induced diabetes or hypertension are also predisposed to ID $(14,15)$. Rodent models suggest further that high alcohol consumption during pregnancy can compromise iron regulation in the offspring (16). Information about the importance of these prenatal factors has not been widely disseminated to pediatricians, and thus some high-risk babies may be ID for several months before their routine screening exams. Delays in detection are of potential concern because of cautionary reports indicating long-term effects of ID on brain development (17). The following research used a nonhuman primate model to investigate whether maternal stress during pregnancy can undermine a baby's iron status, with cascading consequences on other physiologic systems. It is already known that a significant percentage of even normal infant monkeys undergo a transient ID between 4 and 8 mo of age, the same period as humans $(18,19)$.

Gestational stress when extended or severe can influence many systems, including the growth rates of developing babies (20). Prenatal stress in rodents and primates also adversely affects sensitive brain regions, such as the hippocampus, as well as alters the development of endocrine and immune responses (21). While usually attributed to the placental transfer of stress-responsive adrenal hormones from the maternal to fetal compartment (22), the following study shows there can also be important mediation via a micronutrient pathway.

Iron has numerous metabolic and regulatory actions, affecting cell functioning both through its critical role in oxygen transport and through the facilitation of protein synthesis

Abbreviations: ES, early gestational stress; ID, iron deficiency; LS, late gestational stress; MCV, mean corpuscular volume; NK, natural killer cell; RBC, red blood cells 
within cells (23). It is essential for many immune responses $(24,25)$. Anemic children often have more infections, mount smaller inflammatory responses in tests of cutaneous hypersensitivity, and exhibit deficient proliferative and phagocytic responses in vitro (26-28). To evaluate the functional consequences of ID in the infant monkeys, the maturation of one arm of immunity was assessed. Innate immunity should normally develop quickly in a healthy infant, but we hypothesized it might be poorly established or deteriorate in the context of prenatal stress and ID. Our assays focused on an important iron-sensitive lymphocyte, the NK, which is involved in the defense against viruses and surveillance against mutagenic cells (29).

\section{METHODS}

Subjects. Sixty-four infants were generated under standardized conditions in a large breeding colony of rhesus monkeys (Macaca mulatta), where it was possible to control the diet and living environment (30). The mothers were multiparous between 5 and $15 \mathrm{y}$ of age and time-mated to determine gestation length and schedule the two stress conditions. They were fed a commercial diet with an iron content adequate for a nonpregnant animal $(180 \mathrm{mg} / \mathrm{kg}$ biscuit, PMI Nutrition International, Richmond, IN), but more marginal to meet the iron needs of a gravid and lactating female (31). All procedures were approved by the Animal Care and Use Committee of the University of Wisconsin.

Pregnancy manipulations. To investigate the potential effect of maternal stress, 40 pregnant females were briefly disturbed each day for $6 \mathrm{wk}$ or $25 \%$ of the 24-wk pregnancy, either early or late in gestation, and compared with 24 normal pregnant controls. A 10-min acoustical startle protocol was used $5 \mathrm{~d} / \mathrm{wk}$ to create this moderate stress condition between d 50 and 92 or d 105 and 147 postconception ( $n=11$ and 29 , respectively). During the brief relocation into another room each afternoon, the gravid dam was intermittently aroused by three 1 -s broadcasts of a horn, randomly scheduled by a computer program at $1-4 \mathrm{~min}$ intervals. This type of disturbance is sufficient to increase maternal cortisol levels and the female's arousal reaction continues to be elicited through the 6-wk period (32). Blood samples were collected at 2-wk intervals to verify the stress induction, and cortisol measured by RIA. There were no subsequent manipulations during the last month of pregnancy until the natural birth of the baby, when the neonate was weighed. Small blood samples $(<3 \mathrm{~mL})$ were then collected at 2-mo intervals for the hematological and immune analyses through 6 mo of age.

Hematological and immune measures. Blood was collected from each infant to determine a complete blood count $(\mathrm{CBC})$ and to set up the in vitro cytolytic assays. Briefly, mononuclear cells (MNC) were separated by centrifugation on Ficoll-Hypaque (1.077 sg, Sigma Chemical Co., St. Louis, MO), and cultured in wells at five effector:target ratios $(100,50,25,12.5$, and 6.25:1, E:T) for the chromium-51 release cytotoxicity assays. Cytolytic activity was evaluated against three different target cells: Epstein Barr virustransformed cells (Raji), and Burkitt's lymphoma (Daudi), and erythroleukemia (K562) cell lines. Previous research showed that Daudi and Raji targets provide a better index of NK function in monkeys, whereas CD3+CD8+ cytolytic T cells partly contribute to the killing of K562 targets by NK cells (33). After a 4-h incubation, supernatants were harvested and the amount of released radiolabel determined on a gamma counter. Lysis was calculated with the following formula where $\mathrm{x}$ is the experimental data point: \% cytotoxicity $=(\mathrm{x}-$ spontaneous release $) /($ maximum - spontaneous release $) \times$ 100. Spontaneous and maximal lysis were quantified by incubating target cells with either media or $4 \%$ cetrimide, respectively. For illustrative purposes, only lytic data from the 100:1 ratio have been shown. NK activity was clearly developed by 2 mo of age, which enabled us to discern the possible emergence of a negative effect of ID on an established immune response through 6 mo of age.

Immunophenotyping and flow cytometry techniques were used to quantify the number of T lymphocytes and NK cells in circulation at each age point. MAb aimed at two NK surface makers [CD16 (anti-Leu-11a) and CD56 (Leu-19)], several T subsets [CD4 (Leu-3a), CD8 (Leu 2a), Becton Dickinson, Franklin Lakes, NJ], and pan T cells (CD2, 9.6 clone, New England Nuclear Corp., Boston, MA)]; CD3 (FN18, TNO Institute of Applied Radiobiology and Immunology, Rijswijk, The Netherlands). Secondary labels were either fluorescein-conjugated (Becton Dickinson) or phycoerythrin-conjugated (Sigma Chemical Co.) goat antimouse antibodies. Cells were fixed in $1 \%$ paraformaldehyde, and analyzed on a Coulter EPICS flow cytometer (Beckman Coulter, Inc., Fullerton, CA).

Statistical analyses. The effect of prenatal conditions on each hematological and immune parameter was examined initially with ANOVA considering age at assessment as a repeated measure. Bivariate correlations were also computed. Then, the relationships among growth parameters, hematological status and NK activity were evaluated with two-level hierarchical co-varied rate of change models (34). The MCV was used as the RBC measure, and lysis of Raji target cells used to index NK activity. Level 1 included 192 measures of MCV and lysis, whereas level 2 was comprised of the 64 monkeys. Values at 2 mo of age provided the initial data point for the MCV and NK lysis, whereas physical growth was considered in terms of both birth weight and weight gain between birth and 2 mo. These rate-of-change models distinguished between NK changes due to stable variation across individuals, differences in the amount of change over time, and month-to-month fluctuation within individuals after accounting for developmental change. The coefficients conceptually represent: 1) trait levels at the 2-mo assessment, 2) systematic change over time (i.e. slope), and 3) state or month-to-month fluctuations. The analysis specifically tested whether individual fluctuations in ID co-varied with the fluctuations in NK responses by assessing correlations in state information.

\section{RESULTS}

Maternal stress. Blood samples collected at 2-wk intervals indicated that the experimental manipulation of the pregnant females was moderately stressful. Both early and late stress females had significantly elevated cortisol at the end of the afternoon sessions $\left(\mathrm{F}_{2,61}=9.71, p<0.001\right)$ (Fig. 1). Cortisol increments were larger later in pregnancy, in keeping with rising cortisol levels across gestation. However, the magnitude of the mother's hormone response did not correlate with the effect on the infants' iron or immune status, and thus was not included in the statistical modeling of postnatal influences.

Neonatal status. The occurrence of daily maternal disturbance for $25 \%$ of the pregnancy did not have an adverse effect on reproductive success. All 64 infants were delivered vaginally, without human assistance, and reared naturally by their mothers. Birth weights were not reduced significantly by prenatal stress, although the mean weight of infants from the ES pregnancies was lower (Table 1). Gestation lengths were also similar across conditions, with a tendency toward a modest $1-3 \mathrm{~d}$ increase in the disturbed pregnancies $\left(\mathrm{F}_{2,58}=3.09, p<\right.$ $0.053)$. In general, birth weights were correlated with gesta-

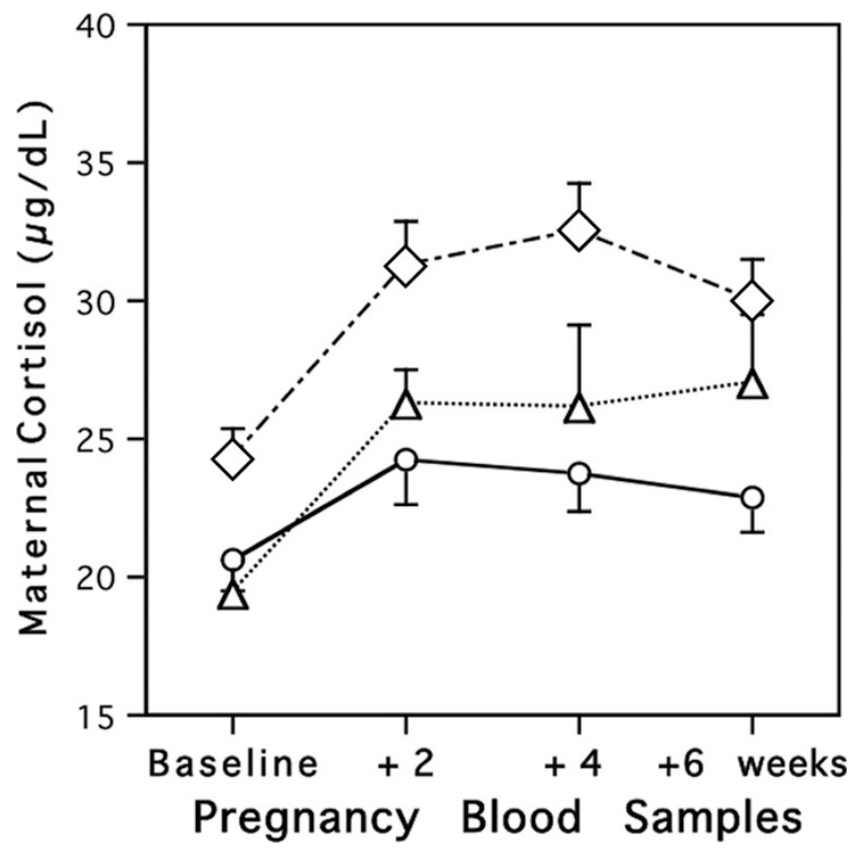

Figure 1. Plasma cortisol levels in pregnant females obtained before and at 2-wk intervals after the initiation of stress manipulations either early (ES: open triangles, $n=11$ ) or late in gestation (LS: open diamonds, $n=29$ ). Blood was collected immediately at the end of the 10-min startle session and compared with cortisol levels in undisturbed control females (C: open circles, $n=24)$. 
Table 1. Birth weights and gestation lengths for infant monkeys from control, ES, and LS pregnancy conditions

\begin{tabular}{lcccc}
\hline & $\begin{array}{c}\text { Control } \\
(n=24)\end{array}$ & $\begin{array}{c}\text { ES } \\
(n=11)\end{array}$ & $\begin{array}{c}\text { LS } \\
(n=29)\end{array}$ & $p$ \\
\hline Birth weight (g) & $507.4(13.8)$ & $474.5(17.4)$ & $500.9(10.2)$ & NS \\
Gestation length (d) & $167.3(0.9)$ & $170.5(1.7)$ & $168.1(0.8)$ & $<0.053$ \\
\hline
\end{tabular}

tional age across the whole sample $(r=0.29, p<0.03)$, which made the combination of a smaller size and a slightly longer pregnancy for the ES neonates appear distinctive.

Hematological profile and NK activity. Maternal stress during pregnancy had a marked effect on the hematological profiles across the first 6 mo of life (Fig. 2). Reflecting the normal demand for iron in a growing baby, the MCV underwent a general decline with age. However, the decreases were significantly greater in the prenatally disturbed infants, and more offspring from ES and LS pregnancies showed a pronounced decrement $\left(\chi^{2}[1]=4.17, p=0.04\right)$. At 6 mo of age, $36 \%$ of the ES and $21 \%$ of the LS infants were sufficiently ID to meet the hematological criteria for anemia in monkeys compared with only $8 \%$ of control infants $(\mathrm{MCV}<60 \mathrm{fL}$, $\mathrm{Hct}<34.9 \%, \mathrm{Hb}<11 \mathrm{~g} / \mathrm{dL})(1)$.

When the infants' lymphocyte responses were examined, prenatal conditions also significantly impacted the capacity to lyse target cells (Fig. 3). Cytolytic activity was well established by 2 mo of age, but the infants from disturbed pregnancies, especially the ES condition, had lower killing across all three cell targets $\left(\mathrm{F}_{2,61}=4.03, p<0.023\right)$. The differences became more striking by 6 mo of age with the emergence of ID. At this age, cytolytic activity against Raji and Daudi cells was further reduced in the ES infants, and marginally lower against K562 cells, whereas most control infants were showing maturational increases in killing. The differential magnitude of the effect across the three cell targets resulted in a significant interaction between prenatal condition and type of target $\left(\mathrm{F}_{4,122}=3.18, p<0.016\right)$.

These differences in cytolytic activity were not due to a lymphocytopenia or a reduction in specific cell subsets. Numbers and percentages of $\mathrm{T}$ lymphocytes and NK cells did not differ in infants from the three pregnancy conditions. The mean percentage of CD16+CD56+ cells averaged between 13.9 and 18.9 and did not change significantly across this age period.
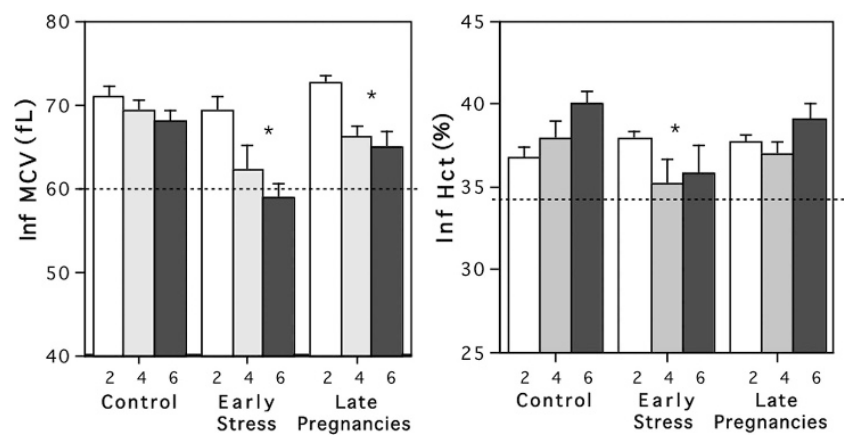

Figure 2. Hematological profiles at 2-mo intervals showing that infants from disturbed pregnancies $(n=40)$ were more likely to become ID by 6 mo of age, especially after stress earlier in gestation $(n=11)$. Declines in MCV and Hct were significantly greater in prenatally stressed infants $\left(\mathrm{F}_{4,122}=2.98\right.$, $p<0.022 ; \mathrm{F}_{4,122}=2.75, p<0.031$, respectively). In contrast to the normal maturational rise in Hct, ES infants showed a decline. Hb levels were also lower and the numbers of the small-sized RBC were higher. By 6 mo of age, $\mathrm{Hb}$ averaged 12.83 in controls versus 12.34 and $11.45 \mathrm{~g} / \mathrm{dL}$ in infants from LS and ES pregnancies, respectively $\left(\mathrm{F}_{4,122}=2.27, p<0.064\right)$. Dotted lines indicate clinical criteria for anemia in monkeys.

Statistical modeling of ID and immune changes. To test the causal nature of the temporal association between the emergence of ID and lower NK lytic activity, more sophisticated statistical modeling was used. These analyses simultaneously evaluated variation in values across individuals and conditions, and the slopes of developmental change $(34,35)$. Table 2 shows that a substantial percentage of variability was due to trait differences between infants, a more modest though still significant percentage was derived from systematic change over time, and finally that the majority of variance in $\mathrm{MCV}$ and lytic activity was attributable to their associated fluctuations. At the trait level, MCV values were correlated with lysis of Raji $(r=0.44$ for level and 0.31 for slope, $p<0.01)$ and Daudi cells $(r=0.47$ for level and $r=0.47$ for slope, $p<$ 001), concurring with the finding that infants with low MCV had lower lysis. The slope of the MCV decline was correlated with Raji $(r=0.41$ for level and $r=0.45$ for slope, $p<$ 0.001 ), Daudi ( $r=0.29$ for level and $r=0.29$ for slope, $p<$ $0.02)$, and K562 ( $r=0.41$ for level and 0.40 for slope, $p<0.001$ ), indicating that a large change in MCV was associated with the NK decrement at 6 mo of age.

Both birth weight and rate of postnatal growth were entered into the hierarchical models to consider their influence on the
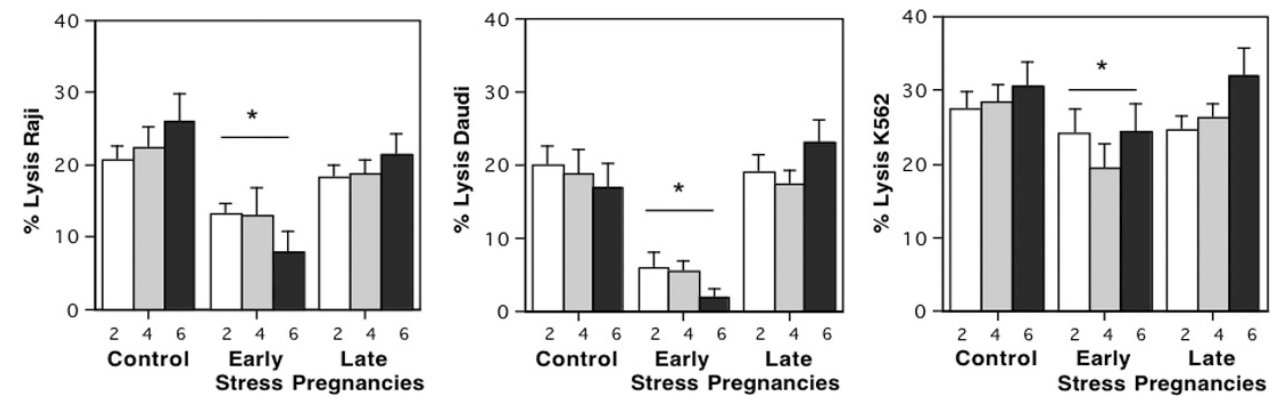

Figure 3. NK activity against three cell targets (Raji, Daudi, K562) was established by 2 mo of age, but declined and became aberrant after emergence of ID in prenatally stressed infants $(n=40)$. Overall, ES infants $(n=11)$ had the lowest cytolytic activity $\left(\mathrm{F}_{2,61}=6.77, p<0.003\right)$. Separate analyses by target cell indicated this prenatal effect was primarily on killing of Daudi and Raji cells $\left(\mathrm{F}_{2,61}=9.24, p<0.001 ; \mathrm{F}_{2,61}=6.32, p<0.004\right.$, respectively). The immune impact of prenatal stress was compounded by the infants' worsening iron status. Shading code: open bars $=2$ mo, shaded bars $=4$ mo, black bars $=6$ mo. 
Table 2. Random effects in the HLM analyses indicating the percentage variance (\%) due to between individual (trait), systematic change over time (slope) and within individual or unsystematic change over time (state) sources of variation

\begin{tabular}{|c|c|c|c|c|c|c|}
\hline & \multicolumn{2}{|c|}{ Between individual (trait) } & \multicolumn{2}{|c|}{ Change over time (slope) } & \multicolumn{2}{|c|}{ Within individual (state) ${ }^{1}$} \\
\hline MCV & 13.74 & $41.50^{\S}$ & 3.48 & $10.52^{\S}$ & 15.88 & 47.97 \\
\hline Daudi & 67.19 & $46.94^{\S}$ & 1.62 & 1.13 & 74.32 & 51.92 \\
\hline K562 & 6.20 & 5.30 & 8.58 & $7.34^{\dagger}$ & 102.12 & 87.36 \\
\hline
\end{tabular}

MCV levels were consistently different between individuals and the change in MCV was moderately stable over time. Levels of Raji lysis were moderately stable within individuals and showed consistent developmental trajectories over time. Variation in levels of Daudi killing was consistent across individuals, but the developmental trajectory was less systematic. Level of K562 killing varied the most across individuals, but some of the developmental change over time was stable within an individual. Therefore, the Raji values were used for evaluating overall associations between growth, iron status and immunity (Fig. 4).

${ }^{1}$ Inferential statistics for within individual variance estimates are not available, although they were substantial

${ }^{*} p<0.05 ;{ }^{\dagger} p<0.001,{ }^{\S} p<0.0001$.

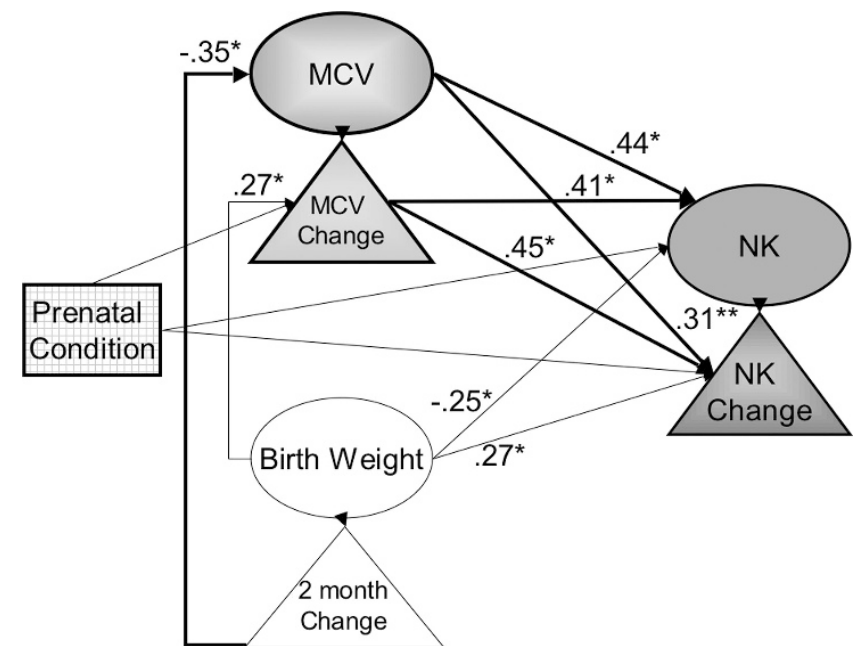

Figure 4. Hierarchical co-varied rate of change models indicated that developmental changes in hematological status were predictive of both lower NK responses and the slope of the decline in lysis. In addition, a lower birth weight in conjunction with faster postnatal growth during the first 2 mo of life augmented demands on iron and contributed significantly to the relationship between RBC and NK functioning. Growing infants were challenged to obtain sufficient dietary iron, and prenatal stress predisposed them toward ID. Significant bivariate correlations are shown in the numbers and asterisks accompanying the arrows $(* p<0.05)$.

heme-immune relationships. Figure 4 illustrates that prenatally stressed infants with lower birth weights and larger growth rates during the first 2 mo of life were the ones most likely to manifest large decreases in MCV and NK activity between 2 and 6 mo of age. Even after controlling for this influence of growth, infants with lower trait MCV had reduced cytolytic activity. Similarly, the decline in $\mathrm{MCV}$ continued to correlate with the decrement in NK activity. Moreover, if a decrease in MCV was greater than the individual's initial trajectory predicted, then the lysis was even more impaired (Raji: $r=0.15$; Daudi, $r=0.20$; K562: $r=$ $0.17 ; p<0.04)$. Thus, the analytic model showed that lower birth weights and/or higher rates of postnatal growth contributed to, but did not fully account for, the effect of prenatal stress on the decreases in MCV and NK activity.

\section{DISCUSSION}

These findings demonstrate several novel and important influences of prenatal events on the development of hematological and immune functions in young infants. They also indicate that the transfer and utilization of iron may be a significant mediating process impacted by maternal stress during pregnancy. While it was already known that premature human babies are at higher risk for ID (12), ours is the first study to document an effect of gestational disturbance on later iron status in a full-term baby within the normal weight range. Most prior research on prenatal stress has focused on mediation by maternal and placental hormones (36), whereas the current results support those who have argued for an equally important role of nutritional factors (37). This influence of iron became increasingly evident during the period of rapid postpartum growth, which accentuated the need for iron, and resulted in a significant impact on innate immunity.

The robustness of the temporal association between growth rates, the MCV decline, and NK lysis in the statistical modeling argues against alternative explanations, such as a simple effect on iron content in the breast milk. While it is known that lactoferrin, a form of iron found in milk, can affect the NK cell (38), the low lactoferrin in the blood stream and the fact that the decreased cytolysis was so coincident with the emergence of ID at 2-6 mo of age would make it less likely that lactoferrin was a direct mediator. The decline in lysis was also not due to a methodological artifact of abnormal iron-depleted RBC contaminating the in vitro assays. It is known that the presence of high numbers of RBC in cell cultures can influence NK activity (39), but our use of separated lymphocytes indicated that the reduced functional capacity of the NK cells was already evident at the time of blood collection. Immunophenotyping of the infants' CD16+CD56+ cells also documented that the lower lysis was not due to fewer NK cells in circulation as a consequence of prenatal stress, but rather a reduced functional capacity.

Given the known susceptibility of the anemic host to infectious illness, the compromised immunity of the ID infants would probably be sufficient to hamper their response to bacterial infections of the gastrointestinal tract and respiratory viruses upon exposure. In the controlled, hygienic conditions of a laboratory setting, the occurrence of infections is reduced, but other research has shown that prenatal stress and ID can make infant monkeys more vulnerable when challenged by enteric pathogens such as Shigella (40). Similarly, ID rat pups have decreased peritoneal macrophage and NK cytolytic activity, and a lower resistance to Salmonella typhimurium (41). Moreover, when ID pups were created by provision of a low iron diet to the dams, 
even 3 wk of iron repletion at weaning did not fully restore their capacity to mount normal antibody responses (42).

The iron dependency of the anemia that emerged in our monkey infants should be highlighted. Anemia is a late manifestation of ID, and it became increasingly evident at 6 mo of age. Prior hematological measures obtained earlier at 4 mo were already indicative of a depletion of storage iron and reduced erythropoiesis. In addition to the lower MCV, signs of demand were evident by compensatory increases in the number of small RBC. From clinical interventions with other infant monkeys, we also know that their anemia can be rapidly reversed by injections of iron or more gradually through provision of an iron-fortified diet. Moreover, in ongoing evaluations of pregnant monkeys fed a high iron diet, it appears possible to ameliorate some of the iron-mediated effects of prenatal stress by supplementation. Conversely, the period of deficiency in a weanling monkey can be extended or worsened by a delay in the introduction of solid foods.

The relationship between psychosocial factors, diet, and the "early anemia" of childhood has been the subject of considerable speculation for over 75 y (43), but this type of information is not always incorporated into clinical practice. Many pediatricians do not routinely take prenatal risk factors into consideration, which include premature delivery, low birth weight, and whether the mother was diabetic during pregnancy $(15,44)$. Our research suggests that high levels of maternal stress may be another factor of potential concern. Discovering that the postpartum effect was most pronounced when the disturbance occurred early in pregnancy, rather than later when it was more proximal to the highest placental transfer of iron, suggests that changes intrinsic to the fetus were set in motion, which perturbed iron storage and use postpartum. For human babies who might be at similarly high risk for ID, it may be advisable to consider an additional hematological exam before the standard "well baby" visit at 8 mo of age, and to recommend an earlier introduction of iron-rich foods (45).

Note: Based on prior reports on ID in infant monkeys, criterion for anemia was set at a MCV below $60 \mathrm{fL}$ rather than the $70 \mathrm{fL}$ used as the cutoff in adult humans (46).

Acknowledgments. The authors thank Ms. H. Crispen for her invaluable assistance with animal husbandry and sample collection, and Ms. A. Slukvina for conducting the NK assays.

\section{REFERENCES}

1. DeMaeyer E, Adiels-Tegman M 1985 The prevalence of anaemia in the world. World Health Stat Q 38:302-316

2. Allen LH 1997 Pregnancy and iron deficiency: unresolved issues. Nutr Rev 55:91-101

3. Lonnerdal B, Dewey KG 1995 Epidemiology of iron deficiency in infants and children. Annales Nestlé 53:11-17

4. Zetterstrom R 2004 Iron deficiency and iron deficiency anaemia during infancy and childhood. Acta Paediatr 93:436-439

5. Dallman PR 1986 Iron deficiency in the weanling: a nutritional problem on the way to resolution. Acta Paediatr Scand Suppl 323:59-67

6. Oski FA 1993 Iron deficiency in infancy and childhood. N Engl J Med 329:190-193

7. Looker AC, Dallman PR, Carroll MD, Gunter EW, Johnson CL 1997 Prevalence of iron deficiency in the United States. JAMA 277:973-976

8. Yip R 1989 The changing characteristics of childhood iron nutritional status in the United States. In: Filer LJ Jr (ed) Dietary Iron: Birth to Two Years. Raven Press, New York, pp 38-56

9. Brotanek JM, Halterman JS, Auinger P, Flores G, Witzman M 2005 Iron deficiency, prolonged bottle-feeding and racial/ethnic disparities. Arch Pediatr Adolesc Med 159:1038-1042
10. Allen LH 2000 Anemia and iron deficiency: effects on pregnancy outcome. Am J Clin Nutr. 71:1280S-1284S

11. Winzerling JJ, Kling PJ 2001 Iron-dependent erythropoiesis in premature infants measured by blood zinc protoporphyrin/heme. J Pediatr 139:134-136

12. Griffin IJ, Cooke RJ, Reid MM, McCormick KP, Smith JS 1999 Iron nutritional status in preterm infants fed formulas fortified with iron. Arch Dis Child Fetal Neonatal Ed 81:F45-F49

13. Lundstrom U, Siimes MA, Dallman PR 1977 At what age does iron supplementation become necessary in low birth weight infants? J Pediatr 91:878-883

14. Rao R, Georgieff MK 2001 Neonatal iron nutrition. Semin Neonatol 6:425-435

15. Rao R, Georgieff MK 2002 Perinatal aspects of iron metabolism. Acta Paediatr Suppl 91:124-129

16. Connor JR 1994 Iron acquisition and expression of iron regulatory proteins in the developing brain: manipulation by ethanol exposure, iron deprivation and cellular dysfunction. Dev Neurosci 16:233-247

17. Lozoff B, Beard J, Connor J, Felt B, Georgieff M, Schallert T 2006 Long-lasting neural and behavioral effects of iron deficiency in infancy. Nutr Rev 64:S34-S43

18. Bicknese EJ, George JW, Hird DW, Paul-Murphy J, Anderson JA, Roberts JR 1993 Prevalence and risk factors for iron deficiency in anemia in weanling rhesus macaques. Lab Anim Sci 43:434-438

19. Kriete MF, Champoux M, Suomi S 1995 Development of iron deficiency anemia in infant rhesus monkeys. Lab Anim Sci 45:15-21

20. Wadhwa PD, Dunkel-Schetter C, Garite TJ, Porto M, Sandman CA 1993 The association between prenatal stress and infant birth weight and gestational age at birth: a prospective investigation. Am J Obstet Gynecol 169:858-865

21. Weinstock M 2001 Alterations induced by gestational stress in brain morphology and behaviour of the offspring. Prog Neurobiol 65:427-451

22. Takahashi LK 1998 Prenatal stress: consequences of glucocorticoids on hippocampal development and function. Int J Dev Neurosci 16:199-207

23. Beard JL 2001 Iron biology in immune function, muscle metabolism, and neuronal functioning. J Nutr 131:568S-580S

24. Moore LL, Humbert JR 1984 Neutrophil bactericidal dysfunction towards oxidant radical sensitive microorganisms during experimental iron deficiency. Pediatr Res 18:789-794

25. Bowlus CL 2003 The role of iron in $\mathrm{T}$ cell development and autoimmunity. Autoimmun Rev 2:73-78

26. Dhur A, Galan P, Hercberg S 1989 Iron status, immune capacity, and resistance to infections. Comp Biochem Physiol A 94:11-19

27. Chandra RK 2002 Nutrition and the immune system from birth to old age. Eur J Clin Nutr 56:S73-S76

28. Walter T, Olivates M, Pizzaro F, Munoz C 1997 Iron, anemia, and infection. Nutr Rev 55:111-124

29. Gogos CA, Kalfarentzos FE, Zoumbs NC 1990 Effect of different types of total parenteral nutrition on T lymphocyte subpopulations and NK cells. Am J Clin Nutr 51:119-122

30. Price KC, Coe CL, Hyde JS 1999 Matrilineal transmission of birth weight in the rhesus monkey across several generations. Obstet Gynecol 94:128-134

31. Bothwell TH 2000 Iron requirements in pregnancy and strategies to meet them. Am J Clin Nutr 72:257S-264S

32. Coe CL, Kramer M, Czeh B, Gould E, Reeves AJ, Kirschbaum C, Fuchs E 2003 Prenatal stress diminishes neurogenesis in the dentate gyrus of juvenile rhesus monkeys. Biol Psychiatry 54:1025-1034

33. Lubach GR, Coe CL, Karaszewski JW, Ershler WB 1996 Effector and target cells in the assessment of natural cytotoxicity in the rhesus monkey. Am J Primatol 39:275-287

34. Sliwinski MJ, Hofer SM, Hall C, Buschke H, Lipton RB 2003 Modeling memory decline in older adults: the importance of preclinical dementia, attrition, and chronological age. Psychol Aging 18:658-671

35. Kraemer HC, Yesavage JA, Taylor JL, Kupfer D 2000 How can we learn about developmental processes from cross-sectional studies or can we? Am J Psychiatry 157:163-171

36. Seckl JR 1997 Glucocorticoids, feto-placental 11-beta hydroxysteroid dehydrogenase type 2, and the early life origins of adult disease. Steroids 62:89-94

37. Murphy VE, Smith R, Giles WB, Clifton VL 2006 Endocrine regulation of human fetal growth: the role of the mother, placenta, and fetus. Endocr Rev 27:141-169

38. Damiens E, Mazurier J, Yazidi I, Masson M, Duthille I, Spik G, Boilly-Marer Y 1998 Effects of human lactoferrin on NK cell cytotoxicity against haematopoietic and epithelial tumor cells. Biochim Biophys Acta 1402:277-287

39. Shau H, Golub SH 1988 Modulation of natural killer-mediated lysis by red blood cells. Cell Immunol 116:60-72

40. Bailey MT, Lubach GR, Coe CL 2004 Prenatal conditions alter the bacterial colonization of the gut in the infant monkey. J Pediatr Gastroenterol Nutr 38:414-421

41. Hallquist NA, McNeil LK, Lockwood JF, Sherman AR 1992 Maternal-iron deficiency effects on peritoneal macrophage and peritoneal natural-killer-cell cytotoxicity in rat pups. Am J Clin Nutr 55:741-746

42. Kochanowski BA, Sherman AR 1985 Decreased antibody formation in antibody deficient rat pups-effect of iron repletion. Am J Clin Nutr 41:278-284

43. Mackay HM 1928 Anaemia in infancy: its prevalence and prevention. Arch Dis Child 3:117-147

44. Siddappa AM, Georgieff MK, Wewerka S, Worwa C, Nelson CA, Deregnier RA 2004 Iron deficiency alters auditory recognition memory in newborn infants of diabetic mothers. Pediatr Res 55:1034-1041

45. Sherriff A, Emond A, Bell JC, Golding J 2001 Should infants be screened for anaemia? A prospective study investigating the relation between haemoglobin at 8 , 12, and 18 months and development at 18 months. Arch Dis Child 84:480-485

46. Lubach GR, Coe CL 2006 Preconception maternal iron status is a risk factor for iron deficiency in infant rhesus monkeys. J Nutr 136:2345-2349 